\title{
$\mathrm{BMJ}$
}

\section{Effects of acupuncture on rates of pregnancy and live birth among women undergoing in vitro fertilisation: systematic review and meta-analysis}

\author{
Eric Manheimer, research associate, ${ }^{1}$ Grant Zhang, assistant professor, ${ }^{1}$ Laurence Udoff, assistant \\ professor, ${ }^{2}$ Aviad Haramati, professor, ${ }^{3}$ Patricia Langenberg, professor and vice-chair, ${ }^{4}$ Brian M Berman, \\ professor, ${ }^{1}$ Lex M Bouter, professor and vice chancellor (rector magnificus) ${ }^{5}$
}

\begin{abstract}
${ }^{1}$ Center for Integrative Medicine, University of Maryland School of Medicine, 2200 Kernan Drive, Kernan Hospital Mansion, Baltimore, MD 21207, USA

${ }^{2}$ Department of Obstetrics, Gynecology and Reproductive Services, University of Maryland School of Medicine

${ }^{3}$ Department of Physiology and Biophysics and Medicine, Georgetown University School of Medicine, Washington, DC

${ }^{4}$ Department of Epidemiology and Preventive Medicine, University of Maryland School of Medicine

${ }^{5}$ VU University Amsterdam De Boelelaan 1105, 1081

HV Amsterdam, the Netherlands Correspondence to: $E$ Manheimer emanheimer@compmed.umm.
\end{abstract} edu

doi:10.1136/bmj.39471.430451.BE

\section{ABSTRACT}

Objective To evaluate whether acupuncture improves rates of pregnancy and live birth when used as an adjuvant treatment to embryo transfer in women undergoing in vitro fertilisation.

Design Systematic review and meta-analysis.

Data sources Medline, Cochrane Central, Embase, Chinese Biomedical Database, hand searched abstracts, and reference lists.

Review methods Eligible studies were randomised controlled trials that compared needle acupuncture administered within one day of embryo transfer with sham acupuncture or no adjuvant treatment, with reported outcomes of at least one of clinical pregnancy, ongoing pregnancy, or live birth. Two reviewers independently agreed on eligibility; assessed methodological quality; and extracted outcome data. For all trials, investigators contributed additional data not included in the original publication (such as live births). Meta-analyses included all randomised patients.

Data synthesis Seven trials with 1366 women undergoing in vitro fertilisation were included in the meta-analyses. There was little clinical heterogeneity. Trials with sham acupuncture and no adjuvant treatment as controls were pooled for the primary analysis. Complementing the embryo transfer process with acupuncture was associated with significant and clinically relevant improvements in clinical pregnancy (odds ratio 1.65, 95\% confidence interval 1.27 to 2.14 ; number needed to treat (NNT) 10 (7 to 17$)$; seven trials), ongoing pregnancy $(1.87,1.40$ to 2.49; NNT 9 (6 to 15); five trials), and live birth (1.91, 1.39 to 2.64; NNT 9 (6 to 17); four trials). Because we were unable to obtain outcome data on live births for three of the included trials, the pooled odds ratio for clinical pregnancy more accurately represents the true combined effect from these trials rather than the odds ratio for live birth. The results were robust to sensitivity analyses on study validity variables. A prespecified subgroup analysis restricted to the three trials with the higher rates of clinical pregnancy in the control group, however, suggested a smaller non-significant benefit of acupuncture (odds ratio $1.24,0.86$ to 1.77 ).
Conclusions Current preliminary evidence suggests that acupuncture given with embryo transfer improves rates of pregnancy and live birth among women undergoing in vitro fertilisation.

\section{INTRODUCTION}

Some $10-15 \%$ of couples have difficulty conceiving at some point in their reproductive lives and seek specialist fertility treatment. ${ }^{1}$ A commonly used option is in vitro fertilisation, which involves retrieving a woman's egg, fertilising the egg in the laboratory, and then transferring the embryo back into the woman's uterus through the cervix. ${ }^{2}$ This entire process is typically referred to as an in vitro fertilisation "cycle" because it involves several procedures, typically over the course of about two weeks, starting when a woman begins taking drugs to stimulate egg production. In 2003, over 120000 treatment cycles were performed in clinics in the United States. ${ }^{2}$ In 2000, about 200000 babies worldwide were conceived through in vitro fertilisation. $^{3}$

Because each cycle is expensive, lengthy, and stressful, new drugs and technologies have been developed to improve success rates. Progress, however, has been limited. Although use of some procedures, initiated before the cycle, have been shown to improve pregnancy rates in women with a poorer prognosis because of specific conditions (such as surgical treatment for tubal disease, ${ }^{4}$ long term treatment with gonadotrophin releasing hormone agonists for women with endometriosis ${ }^{5}$ ), few adjuvant procedures have been shown to be effective for women in general. One exception is luteal phase support, which has been shown to increase pregnancy rates ${ }^{6}$ and is routinely used.

Acupuncture has been used in China for centuries to regulate the female reproductive system. ${ }^{7}$ Three potential mechanisms for its effects on fertility have been postulated. ${ }^{8}$ Firstly, acupuncture may mediate the release of neurotransmitters,${ }^{9}$ which may in turn stimulate secretion of gonadotrophin releasing hormone, thereby influencing the menstrual cycle, 
ovulation, and fertility. ${ }^{10}$ Secondly, acupuncture may stimulate blood flow to the uterus by inhibiting uterine central sympathetic nerve activity. ${ }^{11}$ Thirdly, acupuncture may stimulate the production of endogenous opioids, which may inhibit the central nervous system outflow and the biological stress response. ${ }^{12}$

We conducted a systematic review and meta-analysis of randomised controlled trials to determine whether acupuncture given with embryo transfer improves the rates of pregnancy and live birth among women undergoing in vitro fertilisation.

\section{METHODS}

Identification of studies

We searched the computerised databases Medline, Embase, Cochrane Central, and the Chinese Biomedical Database from inception to January 2007. We searched the following terms as free text terms and MeSH terms (shown in italics): (acupuncture; acupuncture therapy; auriculotherapy; electroacupuncture; Medicine, Oriental Traditional; Medicine, Chinese Traditional; moxibustion) and (reproductive techniques, assisted; fertilization in vitro; embryo transfer; oocytes; egg collection). We combined this search strategy with a methods filter for clinical trials. ${ }^{13}$

We also searched the proceedings of three major annual conferences on assisted reproduction technology for 2001-6: the American Society for Reproductive Medicine, the European Society for Human Reproduction and Embryology, and the Pacific Coast Reproductive Society. We also scanned reference lists of relevant publications.

Selection criteria, data extraction, and quality assessment We selected randomised controlled trials that compared acupuncture with sham acupuncture or no adjuvant treatment. Because we were evaluating acupuncture as a complement to embryo transfer, we considered only trials in which acupuncture was administered within one day of the procedure, with the objective of improving success rates. Trials that included intracytoplasmic injection of sperm as well as in vitro fertilisation were eligible. We excluded trials that evaluated acupuncture as an alternative to conventional analgesia for egg removal.

For trials to be eligible, we had to be able to extract data on at least one of the following outcomes, as recommended ${ }^{14-17}$ : clinical pregnancy (that is, presence of at least one gestational sac or fetal heartbeat, confirmed by transvaginal ultrasound $\left.{ }^{18}\right)$, ongoing pregnancy (that is, pregnancy beyond 12 weeks of gestation, as confirmed by fetal heart activity on ultrasound), or live birth. We included cross over trials only if relevant outcome data from the first phase (that is, before the cross over occurred) were available. ${ }^{19} \mathrm{We}$ ignored any data after cross over occurred.

We included only trials in which acupuncture involved the insertion of needles into traditional meridian points. The needles could be inserted into tender points in addition to the traditional meridian points, and the needles could also be electrically stimulated. We excluded trials of dry needling or trigger point therapy. We also excluded trials of laser acupuncture and electro-acupuncture without needle insertion because most authorities believe acupuncture involves needle insertion. ${ }^{20}$

We imposed no restrictions on publication type (that is, either full article or abstract) or language of publication.

Two authors (EM and GZ) independently selected articles and extracted data, with disagreements resolved by discussion. We extracted data pertaining to quality of the methods, participants, interventions, and outcomes. Methodological quality of the trials was evaluated with the internal and external validity criteria from the checklist created by the Cochrane menstrual disorders and subfertility group. ${ }^{14} \mathrm{We}$ contacted corresponding authors with specific questions related to the design and outcomes of their trials and asked them to review the information we extracted from their trials and clarify any ambiguities.

\section{Data synthesis and analysis}

The measure of treatment effect was the pooled odds ratio of achieving a clinical pregnancy, ongoing pregnancy, or live birth for women in the acupuncture group compared with women in the control group. The odds ratio is the measure of choice for outcomes in subfertility trials because this measure is more likely to show homogeneity when control rates differ between trials. ${ }^{14}$ To allow for a more clinically relevant interpretation, however, we also calculated pooled rate differences between the acupuncture and control groups and converted these rate differences to numbers needed to treat. For our meta-analyses, we used a random effects model because of the expected heterogeneity of the studies' protocols and settings.

All meta-analyses were based on the number of women randomised (rather than on the number of treatment attempts - that is, cycles of in vitro fertilisation) with the intention to treat approach to analysis. ${ }^{14} 19$ We included randomised women who began the in vitro fertilisation process but did not complete the treatment (that is, had no embryo transfer) in the intention to treat meta-analyses. ${ }^{14-16} 19$ Although inclusion of women without an embryo transfer will tend to underestimate the effect of acupuncture,${ }^{16}$ it is the more conservative and appropriate analytical approach $^{14-16} 19$ because it preserves the groups created by the randomisation and reduces the chance of a type I error. ${ }^{16}$ All trials reported pregnancy outcomes resulting from a single cycle.

\section{Subgroup analyses}

To assess whether treatment effects varied with internal validity of studies we performed six subgroup analyses that evaluated whether any pooled results that were significant in analyses of all the trials remained significant when we restricted them to trials judged adequate on each of six internal validity components most commonly used for evaluating quality of randomised trials. ${ }^{21}$ These components, each evaluated with 
a separate subgroup analysis (prespecified, except where indicated), were concealment of allocation of randomisation sequence, blinding of patients, blinding of physicians (post hoc analysis), loss to follow-up (withdrawal rate above or below 10\% of the study population), intention to treat analysis, and generation of allocation sequence.

We evaluated heterogeneity using the $\mathrm{I}^{2}$ test, ${ }^{22}$ which indicates the proportion of variability across trials not explained by chance alone. If the overall $\mathrm{I}^{2}$ value for all trials was reduced when we separated the trials into subgroups according to control with sham acupuncture and no adjuvant treatment, ${ }^{22}$ then we would use the subgroup results as primary. Otherwise the pooled results from all trials would be used for our primary analysis, but with the results from the two subgroups also presented. A priori, we would not expect important heterogeneity of results based on whether or not a sham acupuncture control group was used because all outcomes are entirely objective (that is, pregnancy and births), and unlikely to be largely affected by expectations and placebo effects. ${ }^{23-25}$

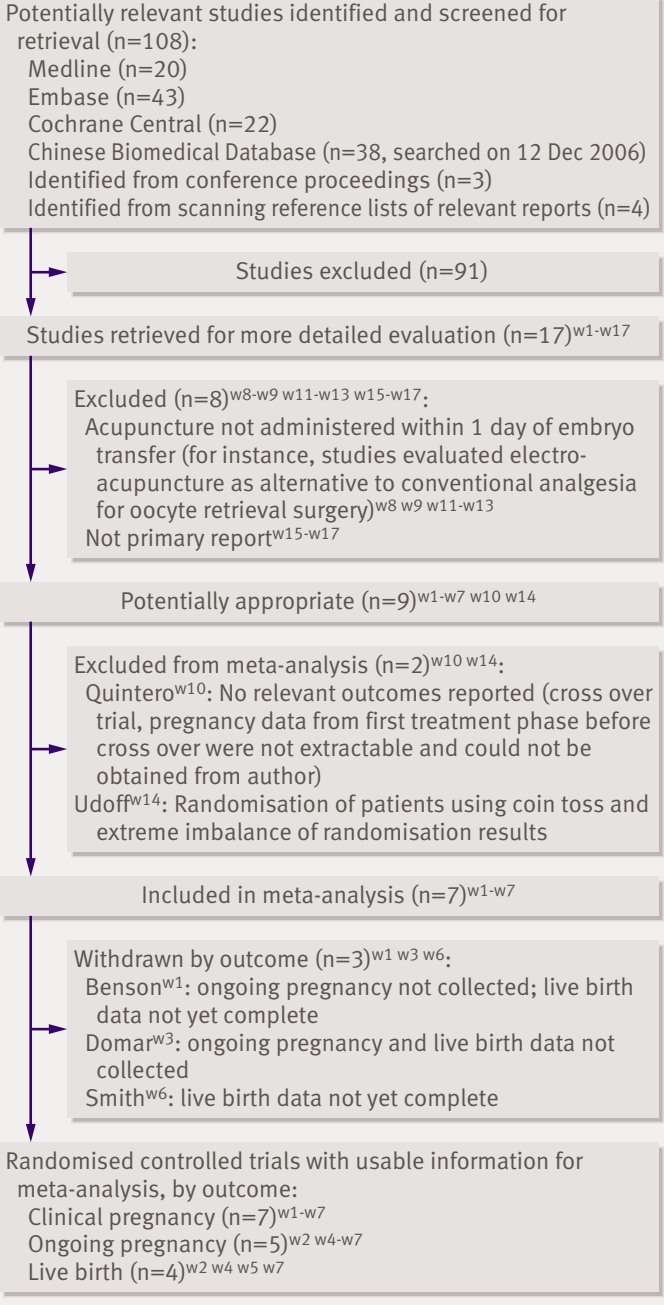

Potentially appropriate $(n=9)^{\text {w1-w7 }}$ w10 w14

Excluded from meta-analysis $(\mathrm{n}=2)^{\mathrm{w} 10}$ w14

Quinterow10: No relevant outcomes reported (cross over trial, pregnancy data from first treatment phase before

$\rightarrow$ cross over were not extractable and could not be obtained from author)

Udoffw14: Randomisation of patients using coin toss and extreme imbalance of randomisation results

Included in meta-analysis $(\mathrm{n}=7)^{\text {w1-w7 }}$

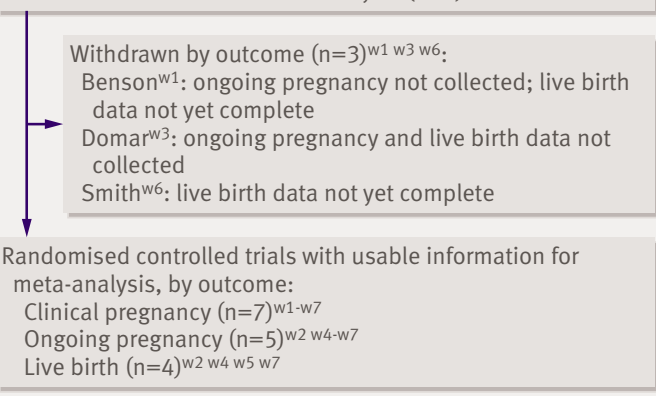

Fig 1 | Flow of studies through selection process
We assessed whether effects of acupuncture varied with three clinical characteristics that might influence success (prespecified, except where indicated): use of extra acupuncture sessions in addition to the sessions before and after the embryo transfer (yes/no) (post hoc analysis); eligibility restricted to women with good quality embryos (yes/no); and low versus high rates of clinical pregnancy in control groups ( $28 \%$ or more, the European average $\left.\mathrm{e}^{26}\right)$. For the subgroup analyses on each of the dichotomised methodological and clinical variables, we also used a significance test, as described by Deeks et al, ${ }^{1927}$ to investigate whether differences in effects of acupuncture between the two subgroups were significant. Subgroup analyses on various clinical characteristics related to the patient (age, cause of diagnosis, duration of infertility, number of previous attempts) were not possible because the trials included typical heterogeneous populations. Other factors (such as electrical stimulation and intracytoplasmic sperm injection) also could not be examined in subgroup analyses because there was little or no heterogeneity across the trials on these factors.

As a sensitivity analysis, we evaluated whether the overall conclusions were affected if we excluded reports published only as abstracts. In addition, in one trial group assignment was unavailable for four randomised patients, none of whom completed the treatment (that is, no embryo transfer). ${ }^{\text {w3 }}$ For our main analysis, we allocated two to the acupuncture group and two to the no adjuvant treatment group. In a posthoc sensitivity analyses, we assumed all four were randomised to the acupuncture group.

\section{RESULTS}

Figure 1 shows details of the selection process. Seven randomised controlled trials with a total of 1366 participants met inclusion criteria (table). ${ }^{\text {wl-w7 }}$ All trials were published in English since 2002, and conducted in four different Western countries. Four were published as full reports ${ }^{\mathrm{w} 2 \mathrm{w} 4 \mathrm{w} 6 \mathrm{w} 7}$ and three as abstracts, ${ }^{\mathrm{w} 1} \mathrm{w}^{\mathrm{w}} \mathrm{w} 5$ in two of the leading reproductive medicine journals. We obtained unpublished methodological information for all seven and unpublished outcome data on live births for three. ${ }^{\mathrm{w} 2 \mathrm{w} 4 \mathrm{w} 5}$

All seven trials used a pragmatic design,${ }^{17}$ including typical clinical populations and using typical interventions before and after randomisation. All included a broad selection of women undergoing in vitro fertilisation, with a wide range of ages, diagnostic categories of infertility, durations of infertility, and numbers of previous treatment cycles. The only difference in the inclusion criteria was that two trials ${ }^{\mathrm{w} 4 \mathrm{w} 5}$ included only women with good quality embryos whereas the five others included women with embryos of varying quality. All trials reported use of intracytoplasmic sperm injection for some women. ${ }^{\text {wl-w7 }}$

The timing of the acupuncture sessions relative to embryo transfer differed somewhat among trials (table). In all trials, however, women received acupuncture immediately before or immediately after the embryo transfer. 
Characteristics of included trials in meta-analysis of studies on acupuncture and in vitro fertilisation

\begin{tabular}{|c|c|c|c|c|c|c|}
\hline Study & No randomised & Acupuncture sessions & Control* & $\begin{array}{l}\text { Allocation } \\
\text { sequence } \\
\text { generation }\end{array}$ & $\begin{array}{l}\text { Allocation } \\
\text { concealment }\end{array}$ & $\begin{array}{c}\text { Baseline } \\
\text { comparability } \dagger\end{array}$ \\
\hline Benson ${ }^{w 1}$ & 103 & $\begin{array}{l}\text { Two sessions: one } 25 \text { minutes before transfer } \\
\text { and one immediately after }(n=53) \ddagger\end{array}$ & No adjuvant treatment $(n=50) \S$ & Adequate & Inadequate & Age only \\
\hline Dieterlew2 $^{w 2}$ & 225 & $\begin{array}{l}\text { Two sessions: one immediately after transfer } \\
\text { and one } 3 \text { days after }(n=116)\end{array}$ & $\begin{array}{l}\text { Needle acupuncture, with needles } \\
\text { inserted in real acupuncture points not } \\
\text { expected to influence fertility }(n=109)\end{array}$ & Adequate & Adequate & Adequate \\
\hline Domar $^{\text {w3 }}$ & 150 & $\begin{array}{l}\text { Two sessions: one } 25 \text { min before transfer and } \\
\text { one immediately after }\left(n=81 \prod\right)\end{array}$ & $\begin{array}{l}\text { No adjuvant treatment (women lay } \\
\text { quietly for same time as those in that } \\
\text { intervention group) }(n=699)\end{array}$ & Adequate & Adequate & Age only \\
\hline Paulus ${ }^{\mathrm{w} 4}$ & 160 & $\begin{array}{l}\text { Two sessions: one } 25 \text { min before transfer and } \\
\text { one immediately after }(n=80)\end{array}$ & No adjuvant treatment $(\mathrm{n}=80)$ & Adequate & Adequate & Adequate \\
\hline Paulus $2003^{\text {w5 }}$ & 200 & $\begin{array}{l}\text { Two sessions: one } 25 \text { min before transfer and } \\
\text { one immediately after }(n=100)\end{array}$ & $\begin{array}{l}\text { Sham needles on real acupuncture } \\
\text { points did not penetrate skin }(n=100)\end{array}$ & Adequate & Adequate & Adequate \\
\hline Smith $2006^{\text {w6 }}$ & 228 & $\begin{array}{l}\text { Three sessions: day } 9 \text { of stimulating injections, } \\
25 \text { min before embryo transfer, and } \\
\text { immediately after }(n=110)\end{array}$ & $\begin{array}{l}\text { Sham needles placed close to, but not } \\
\text { on, real acupuncture points did not } \\
\text { penetrate skin, }(n=118)\end{array}$ & Adequate & Adequate & Adequate \\
\hline Westergaard $^{\text {w7 }}$ & 300 & $\begin{array}{l}\text { Group 1: one session } 25 \text { min before transfer } \\
\text { and one after ( } n=100) \text {; group } 2 \text { : same as group } \\
\text { one, but one additional acupuncture session } \\
\text { two days after transfer }(n=100)^{\star \star}\end{array}$ & No adjuvant treatment $(n=100)$ & Adequate & Adequate†† & Adequate \\
\hline
\end{tabular}

*For all sham controlled trials, sham acupuncture procedure was given on same schedule as that used in true acupuncture group.

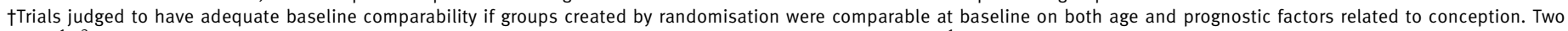
trials $^{\text {w1 }} \mathrm{w}_{3}$ reported only that age of groups were comparable at baseline, as indicated; for one of these trials, ${ }^{\text {w1 }}$ this comparability was based on egg donor age.

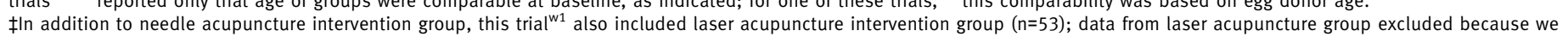
considered only needle acupuncture as an eligible test intervention.

$\S$ Two additional control groups (sham laser and relaxation) excluded from meta-analysis.

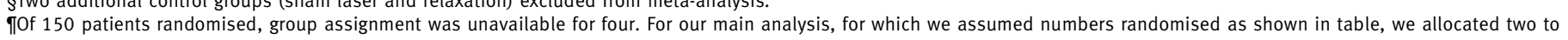
acupuncture group and two to no adjuvant treatment control.

${ }^{\star *}$ Acupuncture groups one and two combined for meta-analysis. ${ }^{50}$

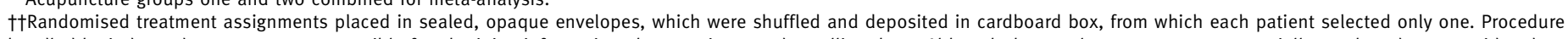

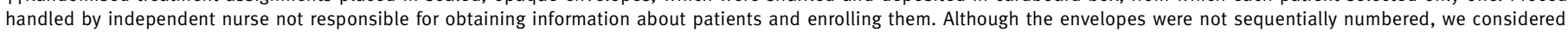

safeguards used in process to have provided adequate assurance of allocation concealment.

In all trials, the acupuncture protocol and selection of acupuncture points was designed for the sole purpose of improving rates of pregnancy. All trials used a fixed selection of acupuncture points for all patients for the sessions before and after embryo transfer. The fixed selection of points for these sessions was similar in all but one trial, ${ }^{\text {w2 }}$ and the points selected were largely based on the points selected in the first published trial that evaluated acupuncture as an adjuvant to embryo transfer. ${ }^{\mathrm{w}}$ Three trials also included one extra acupuncture session, in addition to the sessions before and after the embryo transfer. ${ }^{\mathrm{w} 2 \mathrm{w} 6 \mathrm{w} 7}$ Six trials used ear acupuncture as a supplement to body acupuncture, ${ }^{\text {wl-w6 }}$ and one of these stimulated the true and sham ear acupuncture points using a Chinese herb rather than a needle. $^{\text {w2 }}$

In all the trials the acupuncture sessions lasted 25-30 minutes. Five trials reported that the "de qi" needling sensation was sought, ${ }^{\text {w2 w4-w7 }}$ whereas the two others did not report on de qi. ${ }^{\text {w1 }}{ }^{\text {w3 }}$ No trial used electroacupuncture.

For all trials, there were no significant differences between the randomised groups in the mean numbers of embryos transferred.

\section{Methodological quality of included studies}

The trials generally had high internal validity, in terms of randomisation procedures (table) and follow-up of participants. For all trials but two, ${ }^{\text {w2 }}{ }^{\text {w3 }}$ investigators confirmed no losses to follow-up (which is usual for in vitro fertilisation cycles $\left.{ }^{28}\right)$. For one of the two trials with drop outs, ${ }^{\text {w2 }}$ these were limited to two women in the sham group with ongoing pregnancies, both of whom we assumed to have had live births. ${ }^{17}$ In two other trials, ${ }^{\mathrm{w} 6 \mathrm{w} 7}$ some randomised women began the in vitro fertilisation process but did not complete the treatment (that is, no embryo transfer); however, as noted, these women were still included in the meta-analyses.

Three of the trials used a sham acupuncture

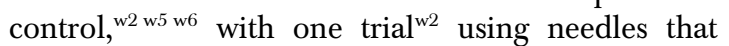
penetrated the skin at acupuncture points selected not to influence fertility ${ }^{29}$ and two ${ }^{\mathrm{w} 5}{ }^{\mathrm{w} 6} \mathrm{using}$ non-penetrating sham needles. For the four other trials, ${ }^{\text {w1 w3 w4 w7 }}$ women in the control group received no adjuvant treatment (table).

Six trials ${ }^{\mathrm{w1}-\mathrm{w} 4 \mathrm{w} 6 \mathrm{w} 7}$ reported their source of funding: three were funded by the in vitro fertilisation clinic, ${ }^{\text {w1 }}{ }^{\mathrm{w} 2} \mathrm{w} 4$ one by government support, ${ }^{\mathrm{w} 7}$ one jointly by clinic and university support, ${ }^{\text {w6 }}$ and one by a company that manufactures fertility drugs. ${ }^{\text {w3 }}$ Four trials did not report on calculation of sample size. ${ }^{\text {w1 w3-w5 }}$

\section{Efficacy analysis}

Our primary analysis is based on results from all included trials because dividing trials according to control group (sham acupuncture and no adjuvant care) increased, rather than reduced, heterogeneity. Embryo transfer with acupuncture was associated with a higher pooled odds for clinical pregnancy $(1.65,95 \%$ confidence interval 1.27 to 2.14 ), ongoing pregnancy 
(1.87, 1.40 to 2.49$)$, and live birth $(1.91,1.39$ to 2.64$)$ (fig 2). The pooled rate differences were 0.11 (0.06 to $0.16)$ for clinical pregnancy, $0.12(0.07$ to 0.17$)$ for ongoing pregnancy, and $0.12(0.06$ to 0.18$)$ for live birth. The numbers needed to treat (rounded up to the next whole number, as recommended $\left.{ }^{31}\right)$ were 10 (7 to 17) for clinical pregnancy, 9 (6 to 15) for ongoing pregnancy, and 9 (6 to 17) for live birth. For the clinical pregnancy outcome, $\mathrm{I}^{2}$ values were $16 \%$ and $4 \%$ for the odds ratio and rate difference effect measures, respectively. All of the heterogeneity was caused by a single trial, ${ }^{\text {w3 }}$ which reported only the clinical pregnancy outcome.

Of the nine subgroup analyses (seven prespecified) on clinical and methodological variables, only the subgroup analysis on the rates of clinical pregnancy in the control group showed a significant effect modification $(\mathrm{P}=0.04)$. Restriction to the three trials with the higher rates of clinical pregnancy in the control group suggested a smaller non-significant benefit of acupuncture (odds ratio $1.24,0.86$ to 1.77 ). No other subgroup restriction resulted in a change to a non-significant effect.

There were no significant adverse effects of acupuncture reported in the two trials that reported on this outcome. $^{\text {w2 w6 }}$

\section{DISCUSSION}

Although still somewhat preliminary, this review suggests that acupuncture given with embryo transfer improves rates of pregnancy and live birth among women undergoing in vitro fertilisation. The strengths of this review include the number of trials and their relatively large sample sizes; pooled odds ratios that are highly significant and clinically important; fairly consistent effect sizes across trials; homogeneity of the acupuncture protocols; use of objective and clinically relevant outcomes; adherence to the intention to treat approach for all meta-analyses; and overall high validity of the trials, as well as robustness of the results to sensitivity analyses on the effects of study validity variables. Because we obtained additional information for all of the trials, the review extends beyond the individual trial publications. For example, we included outcome data on live births from three trials, none of which had those data available when the studies were published.

\section{Methodological strengths and limitations of included trials} The included trials generally had sound methods. Although some used suboptimal methods of concealment of allocation to treatment, blinding ${ }^{2930}$ and intention to treat analyses, ${ }^{28}$ the designs were adequate overall, and any minor design concerns would not be expected to result in a substantial risk of bias. In terms of randomisation, six out of the seven trials ${ }^{\mathrm{w} 2-\mathrm{w} 7}$ used an allocation procedure that would be considered as

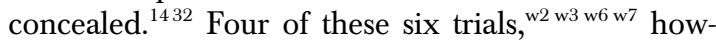
ever, concealed allocation by using sealed envelopes managed by clinical investigators. This is not ideal because of the greater potential for subversion and errors than use of off site treatment allocation. ${ }^{33}$

As for blinding, three trials ${ }^{\mathrm{w} 2}$ w5 6 used a sham control and four ${ }^{\mathrm{w} 3} \mathrm{w} 4 \mathrm{w} 7$ did not blind women to treatment assignment. The necessity to blind participants, however, is arguable when the outcomes are entirely objective (that is, pregnancy and birth), such as in these trials. ${ }^{24}$ It seems unlikely that a woman's knowledge of whether or not she was receiving acupuncture would affect her ability to become pregnant. If the increases in pregnancies in this metaanalysis were due to expectation or placebo effects, then we would also have expected to see a smaller or no effect of acupuncture relative to sham treatment. The effects of acupuncture, however, were the same, regardless of whether acupuncture was compared with sham or no treatment. In addition, an indirect comparison of the proportions of pregnancies pooled from each of the two control arms did not indicate that women allocated to sham acupuncture were more likely to get pregnant compared with women in the no treatment control groups. This indirect comparison is of limited value, however, because it does not maintain the randomisation and therefore has all the limitations of observational data.

A recent methodological study compared the placebo effects of sham acupuncture with the placebo effects of oral pills and showed that patients receiving sham acupuncture can report significantly greater decreases in pain and severity of symptoms than patients receiving oral placebo pills. ${ }^{25}$ While this study found that acupuncture can have an enhanced placebo effect for subjective outcomes, it also found that the placebo effects (of both the sham acupuncture and oral placebo pills) were irrelevant for entirely objective outcomes. Irrelevance of shams for objective outcomes is also supported by recent meta-epidemiological data, which suggest that failure to double blind is associated with exaggerated treatment estimates, but only for trials with subjective outcomes. ${ }^{23}$ Finally, a systematic review has also shown that a placebo can result in a benefit over a "no treatment" control in studies with subjective outcomes such as pain, but that the placebo has no significant effect in studies with objective or binary outcomes. ${ }^{34}$

Blinding of physicians performing the embryo transfer is another potential source of bias (performance bias), and three of the seven included trials did not blind the physicians. ${ }^{\text {w1 w6 w7 }}$ Considering the cost of embryo transfer and the importance of successful transfers to maintaining high pregnancy rates at clinics, we think that physicians would be motivated primarily to perform a successful procedure for all patients, rather than to show that acupuncture, a non-proprietary treatment, is an effective adjuvant procedure. In subfertility trials in general, where outcomes are entirely objective, blinding of either patients or physicians is "infrequently attempted,"17 and such blinding components are not always considered as critical elements related to the evaluation of risk of bias. $^{35}$ 
For all trials but one, ${ }^{\text {w3 }}$ the data were reported in sufficient detail to allow us to conduct full intention to treat analyses for clinical pregnancies. For one other trial, ${ }^{\text {w7 }}$ intention to treat analyses were not used in the analysis for publication, but we could extract the relevant data for our meta-analysis. In this trial, ${ }^{\text {w7 }}$ the patients were randomised too early (at the point of oocyte retrieval), resulting in some women who did not undergo embryo transfer because they had a failure of fertilisation or poor embryo development. These women were excluded from the investigators' analyses, but we included them in our meta-analysis.

Limitations of the systematic review and meta-analysis Limitations of the meta-analysis include heterogeneity of baseline rates across trials, as well as the potential for publication and orientation biases. ${ }^{36}$ Substantial heterogeneity of baseline rates is indicated by the fact that the rates of pregnancy in the control group differed across trials by a factor of three. This heterogeneity is probably not caused by differential selection of patients across trials because each trial was pragmatic, including typical clinic patients with minimal inclusion or exclusion criteria applied. Also, the mean age of the women, an important predictive prognostic variable, ${ }^{17}$ was similar across trials, although one abstract did not report mean age. ${ }^{\mathrm{wl}}$ While distribution of other factors - such as diagnostic category of infertility, fertilisation procedure (in vitro fertilisation or intracytoplasmic sperm injection), and number of previous cyclesvaried somewhat across trials, as expected by chance, such factors have not been shown to be strong predictors of pregnancy rates ${ }^{17}$ and none of these factors seemed to correlate well with the variability in baseline rate. The heterogeneity in baseline rate might more likely be explained by geographical differences in the nature of the typical in vitro fertilisation procedures used. For example, different countries have different regulations for the maximum number of embryos that can be transferred ${ }^{132637}$ and the legality of selecting only high quality embryos, ${ }^{1537}$ and these regulations influence success rates (as well as the rates of multiple pregnancy). ${ }^{137}$ In this review, the country of the trial seemed to be a determinant of the success rates of pregnancy in the control group. ${ }^{3738}$ Because of the heterogeneity in baseline rate, the pooled estimates should be interpreted with caution and might not be directly applicable to any specific clinical population. We pooled the studies' results because of the consistent effect of acupuncture across trials (with one exception $^{\mathrm{w} 3}$ ) on both the odds ratio and rate difference measures, as well as because of the relative homogeneity of the interventions.

As with any systematic review, we cannot exclude the possibility that publication bias might have affected our results. Although we conducted extensive searches to identify relevant studies and funnel plots did not suggest that there were small studies with negative results that were unpublished or not identified, we cannot rule out publication bias and this must be acknowledged as a potential limitation.
Another limitation is the possibility of "hypothesis" or "orientation" bias, which has been described as a type of interpretive bias $^{36}$ in which the orientation or enthusiasm of the investigator for a treatment can be an unintentional determinant of a study's results. ${ }^{36}$ The nature of this research ${ }^{36}$ might have increased the investigators' enthusiasm or conviction of the benefits of the treatments investigated. On the other hand, a primary reason for orientation bias is widely acknowledged to be competing financial interests, ${ }^{3639}$ and as acupuncture is a non-proprietary treatment these might be reduced. We cannot exclude an effect, however, because if acupuncture were included as an adjuvant to in vitro fertilisation, clinics would be able to charge extra for acupuncture and thereby increase profits. While orientation bias cannot be excluded, the potential for such bias is also likely to be minimised in these trials because of the objective outcome measures and the methodologically sound study designs.

A final potential limitation is the inclusion of three studies presented only as abstracts, ${ }^{\text {w1 w3 w5 }}$ which may reflect premature analyses that were not peer reviewed. The corresponding authors, however, provided extensive additional information about their methods and results, beyond what was presented in the three abstracts, which increases our confidence in these studies.

\section{Clinical implications}

The odds ratio of 1.65 suggests that acupuncture increased the odds of clinical pregnancy by $65 \%$ compared with the control groups. It is important to note, however, that the odds ratio significantly overestimates the rate ratio in this context, in which the event (pregnancy) is relatively frequent. In absolute terms, the number needed to treat was 10 , suggesting that 10 patients would need to be treated with acupuncture to bring about one additional clinical pregnancy. These are clinically relevant benefits. ${ }^{16}$ The subgroup analysis restricted to three trials with the higher pregnancy rates at baseline ${ }^{\mathrm{w} 1 \mathrm{w} 3 \mathrm{w} 5}$ suggested a smaller non-significant benefit of acupuncture. A possible explanation for this non-significant finding is that in in vitro fertilisation settings, where the baseline pregnancy rates are already high, the relative added value of additional cointerventions, such as acupuncture, may be reduced. The dependency of the magnitude of the effect of acupuncture on the baseline pregnancy rate warrants further study.

Safety and costs are other considerations. Two large prospective surveys of practitioners show that serious adverse events after acupuncture are rare. ${ }^{4041}$ Among women in labour ${ }^{424}$ and women at various stages of pregnancy, ${ }^{44-46}$ systematic reviews and randomised trials have shown acupuncture to be safe, although limited sample sizes preclude definitive conclusions. The effects of acupuncture in early pregnancy on complications later in pregnancy and on perinatal and infant outcomes have also been investigated in one trial, and no safety concerns were detected. ${ }^{47}$ In vitro fertilisation is an expensive procedure, costing an 
Study

Acupuncture Control

Clinical pregnancy

Sham acupuncture control:

Dieterle $2006^{\text {w2 }} \quad 39 / 116 \quad 17 / 109$

Smith $2006^{\text {w6 }} \quad 34 / 110 \quad 27 / 118$

Paulus $2003^{\text {w5 }} \quad 43 / 100 \quad 37 / 100$

Subtotal $(95 \% \mathrm{Cl})$

$326 \quad 327$

Total events: 116 (acupuncture), 81 (control)

Test for heterogeneity: $\left.\right|^{2}=37.6 \%$

Test for overall effect: $z=2.39, P=0.02$

No adjuvant treatment control:

Benson 2006 ${ }^{\mathrm{w} 1} \quad 29 / 53 \quad 22 / 50$

Domar $2006^{\text {w3 }} \quad 24 / 81 \quad 22 / 69$

Paulus $2002^{\text {w4 }} \quad 34 / 80 \quad 21 / 80$

Westergaard 2006 ${ }^{\mathrm{w} 7} \quad 70 / 200 \quad 21 / 100$

Subtotal $(95 \% \mathrm{Cl}) \quad 414$

Total events: 157 (acupuncture), 86 (control)

Test for heterogeneity: $I^{2}=23.2 \%$

Test for overall effect: $z=2.41, P=0.02$

\section{Total (95\%)}

$740 \quad 626$

Total events: 237 (acupuncture), 167 (control)

Test for heterogeneity: $I^{2}=16.0 \%$

Test for overall effect: $z=3.74, P=0.0002$

\section{Ongoing pregnancy}

Sham acupuncture control:

$\begin{array}{lll}\text { Dieterle } 2006^{\text {w2 }} & 33 / 116 & 15 / 109 \\ \text { Smith } 2006^{\text {w6 }} & 31 / 110 & 22 / 118 \\ \text { Paulus 2003 } & 35 / 100 & 26 / 100\end{array}$

Subtotal $(95 \% \mathrm{Cl})$

Total events: 99 (acupuncture), 63 (control)

Test for heterogeneity: $I^{2}=0 \%$

Test for overall effect: $z=3.25, P=0.01$

No adjuvant treatment control:

Paulus 2002 ${ }^{\text {w4 }} \quad 26 / 80 \quad 14 / 80$

Westergaard 2006 w7 $\quad 58 / 200 \quad 19 / 100$

Subtotal $(95 \% \mathrm{Cl}) \quad 280 \quad 180$

Total events: 84 (acupuncture), 33 (control)

Test for heterogeneity: $\left.\right|^{2}=0 \%$

Test for overall effect: $\mathrm{z}=2.80, \mathrm{P}=0.005$

\section{Total (95\%)}

$606 \quad 507$

Total events: 183 (acupuncture), 96 (control)

Test for heterogeneity: $I^{2}=0 \%$

Test for overall effect: $z=4.29, P<0.0001$

\section{Live birth}

Sham acupuncture control:

$\begin{array}{lcc}\text { Dieterle } 2006^{\text {w2 }} & 33 / 116 & 15 / 109 \\ \text { Paulus } 2003^{\text {w5 }} & 35 / 100 & 26 / 100 \\ \text { Subtotal }(95 \% \mathrm{Cl}) & 216 & 209\end{array}$

Subtotal $(95 \% \mathrm{Cl}) \quad 216 \quad 209$

Total events: 68 (acupuncture), 41 (control)

Test for heterogeneity: $I^{2}=8.8 \%$

Test for overall effect: $z=2.67, P=0.008$

No adjuvant treatment control:

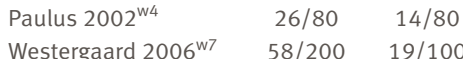

Subtotal $(95 \% \mathrm{Cl}) \quad 280 \quad 180$

Total events: 84 (acupuncture), 33 (control)

Test for heterogeneity: $I^{2}=0 \%$

Test for overall effect: $z=2.80, P=0.005$

Total (95\%)

$496 \quad 389$

Total events: 152 (acupuncture), 74 (control)

Test for heterogeneity: $I^{2}=0 \%$

Test for overall effect: $z=3.95, P<0.0001$

Fig 2 | Effects of acupuncture on clinical pregnancy, ongoing pregnancy, and live birth outcomes $\begin{array}{cc}\text { Weight } & \text { Odds ratio } \\ \text { (\%) } & \text { (random) }(95 \% \mathrm{Cl})\end{array}$

(random) $(95 \% \mathrm{C})$

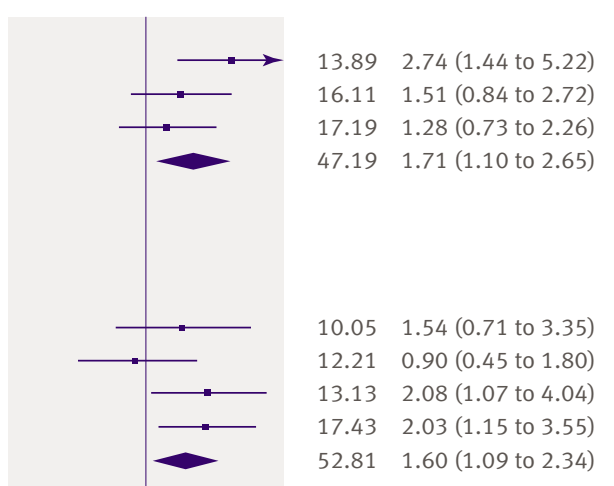
21.171 .71 (0.92 to 3.19) $22.241 .53(0.84$ to 2.81$)$ 61.241 .83 (1.27 to 2.64$)$

$14.862 .27(1.08$ to 4.77$)$ $23.901 .74(0.97$ to 3.13$)$ 38.761 .93 (1.22 to 3.05$)$

100.001 .87 (1.40 to 2.49 )

$22.62 \quad 2.49$ (1.26 to 4.91$)$ 28.211 .53 (0.84 to 2.81 ) 50.831 .91 (1.19 to 3.06)

18.852 .27 (1.08 to 4.77$)$ 30.321 .74 (0.97 to 3.13 ) $49.17 \quad 1.93$ (1.22 to 3.05 )

100.001 .91 (1.39 to 2.64$)$

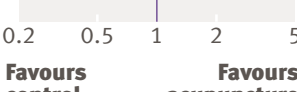

$\begin{array}{lr}\text { Favours } & \begin{array}{r}\text { Favours } \\ \text { control acupuncture }\end{array}\end{array}$
100.001 .65 (1.27 to 2.14 )

17.832 .49 (1.26 to 4.91$)$

\section{WHAT IS ALREADY KNOWN ON THIS TOPIC}

In vitro fertilisation is lengthy, expensive, and stressful

Safe, low cost, adjuvant treatments to improve success rates would benefit patients and reduce costs

\section{WHAT THIS STUDY ADDS}

Current evidence from methodologically sound trials showed an odds ratio of more than 1.6 for clinical pregnancy after in vitro fertilisation with adjuvant acupuncture

On average, 10 women would need to be treated with acupuncture to bring about one additional clinical pregnancy

The magnitude of this effect depended on the baseline pregnancy rate

average of $\$ 12400$ ( $£ 6300, € 8480$ ) per cycle in the United States. ${ }^{48}$ If acupuncture increased the likelihood of success of an individual cycle, then the need for a subsequent cycle would be reduced, and overall costs would be decreased. Even if such increases were small, and, for example, 17 patients needed to be treated with acupuncture to bring about one additional pregnancy (that is, the lowest range of our 95\% confidence interval), an acupuncture cointervention may still be cost effective, considering the negligible costs of two to four sessions of acupuncture, relative to the high costs of in vitro fertilisation.

\section{Conclusion and future research}

Although current estimates of the effects of adjuvant acupuncture on in vitro fertilisation are significant and clinically relevant, they are still somewhat preliminary. Additional randomised trials are needed to quantify findings further and investigate the relation between baseline rate of pregnancy and the efficacy of adjuvant acupuncture. Future trials should adhere to CONSORT guidelines ${ }^{49}$ and guidelines developed specifically for subfertility trials ${ }^{141635}$ and should evaluate an acupuncture protocol similar to that first reported by Paulus and colleagues, ${ }^{\text {w4 }}$ and suggested to have some beneficial effects, according to the trials included in this review.

We thank Laura Benzel and Don Frese, University of Maryland, Baltimore, and Jianping Liu, Beijing University of Chinese Medicine, for assistance with searching for studies. We also thank Jeanette Ezzo, Elizabeth Pradhan, Daniëlle AWM van der Windt, Susan Wieland, and Qi Zhu for useful suggestions during the preparation of the paper, and Kevin Chen for statistical support. Most importantly, we thank Stefan Dieterle, Alice Domar, Wolfgang Paulus, Caroline Smith, Alan Theall (for the Benson et al trial), and Lars Westergaard, who are all coauthors of included randomised controlled trials, for confirming and providing data related to their respective trials.

Contributors: EM and GZ determined inclusion eligibility of trials, extracted data from the trials, and assessed the methodological quality of the trials. $\mathrm{AH}$ provided a third assessment of the methodological quality of all the trials. EM entered and organised the data, conducted the analyses, and drafted the manuscript. EM, GZ, LU, AH, PL, BMB, and LMB all contributed to the conception and design. EM, GZ, LU, AH, PL, and LB contributed to the analysis and interpretation of data. EM, GZ, LU, AH, PL, BMB, and LMB critically revised the article for important intellectual content and approved the final version. EM is guarantor. 
Funding: EM and BMB were funded by grant No R24 AT001293 from the National Center for Complementary and Alternative Medicine (NCCAM) of the US National Institutes of Health.

Competing interests: None declared.

Ethical approval: Not required.

Provenance and peer review: Not commissioned; externally peer reviewed.

1 Evers IL. Female subfertility. Lancet 2002;360:151-9.

2 Centers for Disease Control and Prevention. Assisted reproductive technology success rates: national summary and fertility clinic reports 2003. Atlanta, GA: US Department of Health and Human Services, 2005. www.cdc.gov/art/art2003.

3 Adamson GD, de Mouzon I, Lancaster P, Nygren KG, Sullivan E, Zegers-Hochschild F. World collaborative report on in vitro fertilization, 2000. Fertil Steril 2006;85:1586-622.

4 Johnson NP, Mak W, Sowter MC. Surgical treatment for tubal disease in women due to undergo in vitro fertilisation. Cochrane Database Syst Rev 2004;3:CD002125.

5 Sallam HN, Garcia-Velasco JA, Dias S, Arici A. Long-term pituitary down-regulation before in vitro fertilization (IVF) for women with endometriosis. Cochrane Database Syst Rev 2006;1:CD004635.

6 Daya S, Gunby J. Luteal phase support in assisted reproduction cycles. Cochrane Database Syst Rev 2004;3:CD004830.

7 Maciocia G. Obstetrics and gynecology in Chinese medicine. New York: Churchill Livingstone, 1997.

8 Chang R, Chung PH, Rosenwaks Z. Role of acupuncture in the treatment of female infertility. Fertil Steril 2002;78:1149-53.

9 Mayer DJ, Price DD, Rafii A. Antagonism of acupuncture analgesia in man by the narcotic antagonist naloxone. Brain Res 1977;121:368-72.

10 Ferin M, Vande Wiele R. Endogenous opioid peptides and the control of the menstrual cycle. Eur J Obstet Gynecol Reprod Biol 1984;18:365-73.

11 Stener-Victorin E, Waldenstrom U, Andersson SA, Wikland M. Reduction of blood flow impedance in the uterine arteries of infertile women with electro-acupuncture. Hum Reprod 1996;11:1314-7.

12 Cho ZH, Chung SC, Jones JP, Park JB, Park HJ, Lee HJ, et al. New findings of the correlation between acupoints and corresponding brain cortices using functional MRI. Proc Natl Acad Sci U S A 1998;95:2670-3.

13 Glanville JM, Lefebvre C, Miles JN, Camosso-Stefinovic J. How to identify randomized controlled trials in Medline: ten years on. J Med Libr Assoc 2006;94:130-6.

14 Clarke J, Farquhar C, Prentice A, Barlow D, Moore V, Vail A, et al. Menstrual disorders and subfertility group. About The Cochrane Collaboration (Cochrane Review Groups (CRGs)) 2006, Issue 3. Art. No: MENSTR. www.mrw.interscience.wiley.com/cochrane/clabout/ articles/MENSTR/frame.html.

15 Griesinger G, Dafopoulos K, Schultze-Mosgau A, Felberbaum R, Diedrich $\mathrm{K}$. What is the most relevant standard of success in assisted reproduction? Is BESST (birth emphasizing a successful singleton at term) truly the best? Hum Reprod 2004;19:1239-41.

16 Daya S. Pitfalls in the design and analysis of efficacy trials in subfertility. Hum Reprod 2003;18:1005-9.

17 Arce JC, Nyboe Andersen A, Collins J. Resolving methodological and clinical issues in the design of efficacy trials in assisted reproductive technologies: a mini-review. Hum Reprod 2005;20:1757-71.

18 Zegers-Hochschild F, Nygren KG, Adamson GD, de Mouzon J, Lancaster P, Mansour R, et al. The ICMART glossary on ART terminology. Hum Reprod 2006;21:1968-70.

19 Deeks JJ, Higgins JPT, Altman DG, eds. Analysing and presenting results. In: Higgins JPT, Green S, ed. Cochrane handbook for systematic reviews of interventions 4.2.5. 2005; section 8. www.cochrane.org/resources/handbook/hbook. htm.

20 Birch S. Systematic reviews of acupuncture-are there problems with these? Clin Acupuncture Oriental Med 2001;2:17-22.

21 Moja LP, Telaro E, D’Amico R, Moschetti I, Coe L, Liberati A. Assessment of methodological quality of primary studies by systematic reviews: results of the metaquality cross sectional study. BMJ 2005;330:1053.

22 Higgins JP, Thompson SG, Deeks JJ, Altman DG. Measuring inconsistency in meta-analyses. BMJ 2003:327:557-60.

23 Wood L, Egger M, Gluud LL, Schulz K, Altman D, Juni P, et al. The association of allocation concealment and blinding with estimated treatment effect varies according to type of outcome: a combined analysis of meta-epidemiological studies. 14th Cochrane Colloquium, Dublin, Ireland, October 23-26, 2006: abstract 0-19.
24 Juni P, Altman DG, Egger M. Systematic reviews in health care: Assessing the quality of controlled clinical trials. BMJ 2001;323:42-6.

25 Kaptchuk TJ, Stason WB, Davis RB, Legedza AR, Schnyer RN, Kerr CE, et al. Sham device $v$ inert pill: randomised controlled trial of two placebo treatments. BMJ 2006;332:391-7.

26 Nyboe Andersen A, Gianaroli L, Nygren KG. Assisted reproductive technology in Europe, 2000. Results generated from European registers by ESHRE. Hum Reprod 2004;19:490-503.

27 Deeks JJ, Altman DG, Bradburn MJ. Statistical methods for examining heterogeneity and combining results from several studies in metaanalysis. In: Egger M, Davey Smith G, Altman DG, eds. Systematic reviews in health care: meta-analysis in context . 2nd ed. London: BM Publishing Group, 2001.

28 Collins J. The play of chance. Fertil Steril 2006;85:1364-7.

29 Domar AD. Acupuncture and infertility: we need to stick to good science. Fertil Steril 2006;85:1359-61.

30 Myers ER. Acupuncture as adjunctive therapy in assisted reproduction: remaining uncertainties. Fertil Steril 2006;85:1362-3.

31 In: Alderson P, Green S, eds. Cochrane Collaboration's open learning material for Cochrane reviewers. 2002: module 11-6. www.cochranenet.org/openlearning $/ \mathrm{html} / \bmod 11-6 . \mathrm{htm}$.

32 Higgins I, Green S, eds. Assessment of study quality. In: Cochrane handbook for systematic reviews of interventions 4.2.5. 2005; section 6.2. www.cochrane.org/resources/handbook/hbook. htm.

33 Piantadosi S. Treatment allocation. Clinical trials: a methodologic perspective . New York, NY: John Wiley, 1997:203-29.

34 Hrobjartsson A, Gotzsche PC. Is the placebo powerless? An analysis of clinical trials comparing placebo with no treatment. $N$ Engl J Med 2001:344:1594-602

35 Vail A, Gardener E. Common statistical errors in the design and analysis of subfertility trials. Hum Reprod 2003;18:1000-4.

36 Kaptchuk TJ. Effect of interpretive bias on research evidence. BMJ 2003;326:1453-5.

37 Ludwig M, Schopper B, Katalinic A, Sturm R, Al-Hasani S, Diedrich K. Experience with the elective transfer of two embryos under the conditions of the German embryo protection law: results of a retrospective data analysis of 2573 transfer cycles. Hum Reprod 2000;15:319-24.

38 Pinborg A, Loft A, Ziebe S, Nyboe Andersen A. What is the most relevant standard of success in assisted reproduction? Is there a single "parameter of excellence"? Hum Reprod 2004;19:1052-4.

39 Smith R. Beyond conflict of interest. Transparency is the key. $B M$ J 1998;317:291-2.

40 White A, Hayhoe S, Hart A, Ernst E. Adverse events following acupuncture: prospective survey of 32000 consultations with doctors and physiotherapists. BMJ 2001;323:485-6.

41 MacPherson $\mathrm{H}$, Thomas $\mathrm{K}$, Walters S, Fitter $\mathrm{M}$. The York acupuncture safety study: prospective survey of 34000 treatments by traditional acupuncturists. BMJ 2001:323:486-7.

42 Lee H, Ernst E. Acupuncture for labor pain management: a systematic review. Am J Obstet Gynecol 2004;191:1573-9.

43 Smith CA, Collins CT, Cyna AM, Crowther CA. Complementary and alternative therapies for pain management in labour. Cochrane Database Syst Rev 2006;4:CD003521.

44 Elden H, Ladfors L, Olsen MF, Ostgaard HC, Hagberg H. Effects of acupuncture and stabilising exercises as adjunct to standard treatment in pregnant women with pelvic girdle pain: randomised single blind controlled trial. BMJ 2005;330:761.

45 Wedenberg K, Moen B, Norling A. A prospective randomized study comparing acupuncture with physiotherapy for low-back and pelvic pain in pregnancy. Acta Obstet Gynecol Scand 2000;79:331-5.

46 Kvorning N, Holmberg C, Grennert L, Aberg A, Akeson J. Acupuncture relieves pelvic and low-back pain in late pregnancy. Acta Obstet Gynecol Scand 2004;83:246-50.

47 Smith C, Crowther C, Beilby J. Pregnancy outcome following women's participation in a randomised controlled trial of acupuncture to treat nausea and vomiting in early pregnancy. Complement Ther Med 2002;10:78-83.

48 American Society for Reproductive Medicine. Frequently asked questions about infertility. www.asrm.org/Patients/faqs.html.

49 Altman DG. Better reporting of randomised controlled trials: the CONSORT statement. BMJ 1996;313:570-1.

50 Alderson P, Green S, eds. Cochrane collaboration's open learning material for Cochrane reviewers. 2002: section A2. www.cochrane-net.org/openlearning/HTML/ modA2-5.htm.

Accepted: 17 December 2007 